\title{
Proposta de um Supercapacitor Nanoestruturado
}

\author{
Proposal of a Nanostructured Supercapacitor
}

\author{
André Luiz Ramos Prado ${ }^{1}$ J Jocenir Boita ${ }^{2}$ \\ ${ }^{1}$ Universidade Federal de Santa Maria - Campus Cachoeira do Sul, Cachoeira do Sul, Brasil \\ andreluizramosprado@icloud.com \\ ${ }^{2}$ Universidade Federal de Santa Maria - Campus Cachoeira do Sul, Cachoeira do Sul, Brasil \\ jocenir.boita@ufsm.br
}

\section{Resumo}

Nos estudos em fisica da matéria condensada, à medida que se diminui uma partícula, unicidades se tornam peculiares, podendo até mesmo definir novas propriedades em sua nanoestrutura, sejam essas elétricas, químicas, entre outras (BOITA et al.,2017). Tendo como base de estudo a formação de nanoparticulas (NP's) de óxidos de ferro ( $\left.\mathrm{Fe}_{2} \mathrm{O}_{3}\right)$, que apresentam propriedades eletrônicas muito interessantes, como alterações químicas nas estruturas atômicas sobre interferência do meio externo (KLEM,2017). Pertinentes rendimentos qualitativos e quantitativos sobre capacitância do material, tempo de carga e descarga, eficiência em aplicações com o uso de filmes finos nanoestruturados (KLEM,2017). Por conseguinte, o $\left(\mathrm{Fe}_{2} \mathrm{O}_{3}\right)$ é promissor para nanotecnologia e motiva novas aplicações nas engenharias. De natureza igual tem-se outras nanopartículas, como Paládio (Pd), Platina (Pt) e Cobre (Cu). Este último por ser um excelente condutor de eletricidade, é muito empregado na fabricação de fios e aparelhos elétricos em macro escala. Direcionado por aplicações de armazenamento de energia, analisase as possiveis interações de nanoestruturas metálicas sobre a quantidade de portadores de cargas, utilizando diferenças de potencial sobre um conjunto de placas nanoestruturadas em paralelo (MIN et al.,2017). Desta forma pode-se construir um supercapacitor, com campo elétrico gerado entre as áreas das placas por uma distância mínima e isolada por uma elevada rigidez dielétrica ${ }^{4}$ (ZARBIN, 2017).

Palavras-chave: Nanoparticulas; Nanotecnologia; Supercapacitor

\section{Abstract}

By studies in condensed matter physics, as a particle decreases, unicity's become peculiar, and may even define new properties in their nanostructure, be they electrical, chemical, among others (BOITA et al.,2017). Based on the study of the formation of nanoparticles (NP's) of iron oxides $\left(\mathrm{Fe}_{2} \mathrm{O}_{3}\right)$, which present very interesting electronic properties, such as chemical changes in the atomic structures on external medium interference (KLEM,2017). Therefore, $\left(\mathrm{Fe}_{2} \mathrm{O}_{3}\right)$ is promising for nanotechnology that motivates new applications in the engineering, of equal nature are other nanoparticles, being Pd (Pd), Platinum (Pt), Copper $(\mathrm{Cu})$. The latter as excellent conductor of electricity and therefore is very much employed in the manufacture of wires and electrical appliances in macro scale. Directed by energy storage applications, it is analyzed the possible interactions of metallic nanostructures on the quantity of load carriers, using potential differences on a set of nanostructured plates in parallel (MIN et al.,2017). In this way a supercapacitor can be forged, with an electric field generated between the areas of the plates by a minimum distance and isolated by a high dielectric stiffness (ZARBIN, 2017).. Suitable for qualitative and quantitative results on device capacitance, loading and unloading time, efficiency in applications with the use of nanostructured thin films.

Keywords: Nanoparticles; Nanotechnology; Supercapacitor 


\section{Introdução}

O desenvolvimento proporcionado pela física da matéria condensada, responsável por investigações de sistemas e/ou problemas em nanoescala, tem obtido significativas projeções para o futuro, em expectativa sobre os impactos de que toda matéria é precursora de novos materiais em potencial podendo gerar importantes mudanças sobre a qualidade de vida e na conservação do meio ambiente.

Espera-se que o desenvolvimento da nanotecnologia não se limite na exploração de novos fenômenos e novas teorias do mundo quântico, mas inove as formas de fabricação com tecnologias em nanoescala, dando assim uma nova frente de produção, a dos nanomateriais.

Os problemas de engenharia foram sendo solucionados ao decorrer do desenvolvimento tecnológico, transformando máquinas robustas e de baixa eficiência energética, em sistemas que interligam circuitos eletrônicos simples ou complexos para amortecerem os gastos gerados.

O uso de dispositivos eletrônicos para área da engenharia elétrica é o pilar do desenvolvimento tecnológico, pois, com a crescente demanda de tecnologia, necessita-se desenvolver proporcionalmente os dispositivos que, quando projetados, tornam possíveis os sistemas de controle, análises, comunicações e processamentos.

Nos problemas de engenharia demandam-se, muitas vezes, dispositivos com caráter sumo a dispositivos semelhantes. Os capacitores eletrolíticos, poliéster, cerâmicos, tântalo, mica, SMD's e variáveis são exemplos de dispositivos eletrônicos que necessitam de uma implementação, sendo estes cabíveis e classificados como supercapacitores com capacidades eletrônicas e utilidades ampliadas.

\subsection{Princípios do Capacitor}

Os capacitores, em geral, são complexos devido às diversas formas construtivas, sendo definidos constantemente em conformidade às suas aplicações específicas. Muitos capacitores são classificados pelo uso em circuitos elétricos de corrente e tensão contínua ou alternada, pelos níveis de variações da frequência e período. Porém, sua estrutura física é análoga para todos os tipos de dispositivos de capacitância, baseando-se em elementos condutores e isolantes.

Construído com duas placas paralelas, chamadas de eletrodos, estes responsáveis pelo armazenamento de cargas minoritárias e majoritárias, sendo positivas (prótons) e/ou negativas (elétrons). Os portadores acumulam-se nas áreas de materiais condutores devido a separação por um elemento isolante caracterizado em equações como dielétrico.

Capacitores em geral reagem a uma diferença de potencial aplicada em terminais fundidos às áreas de material condutor, habitualmente eletrodos metálicos, ocasionando cargas de sinais opostos induzidas nas superfícies das placas paralelas até que a diferença de potencial, gerado por uma fonte de tensão (CA-corrente alternada ou DC-corrente continua) entre os terminais, se iguale à da fonte.

Quando a geração de tensão é retirada do sistema (fonte mais capacitor) e o dispositivo é curto-circuitado, os terminais conectados diretamente entre si ou os terminais conectados através de um resistor, forçam o movimento das cargas elétricas de forma a pôr as placas carregadas em equilíbrio eletrostático. Nesse processo, é gerada uma corrente elétrica, liberando uma potência aparente, ativa ou reativa.

Para o capacitor de placas planas e paralelas, a capacitância eletrostática é dada pela razão entre a área (A) dos eletrodos e a distância (d) entre eles, multiplicado pela constante dielétrica $(\varepsilon)$, como na equação (1): 


$$
C=\varepsilon \times \frac{A}{d}
$$

A energia armazenada (E) em um capacitor é dada pela equação (2):

$$
E=\frac{c \times V^{2}}{2}
$$

A energia armazenada em capacitores eletrostáticos é baixa, pois mesmo permitindo a aplicação de alta tensão, a capacitância não excede valores necessários para sistemas eletrônicos embarcados de equipamentos laboriosos, pois não se aplicam a capacitores comuns. Os capacitores eletrostáticos possuem baixa resistência equivalente em série (RES), o que permite a liberação de energia de forma extremamente rápida, ou seja, exibem alta potência $(\mathrm{P})$.

A partir destes conceitos básicos, surge a pretensão de análises de novas características em ligas metálicas e/ou soluções químicas em nanoescala, sendo necessárias para proporcionar o aprimoramento de diversos dispositivos, em particular, dos capacitores e supercapacitores constituídos por nanoestruturas, de modo que fomente novas formas de construção e aplicações de equipamentos eletrônicos suportados pelos dispositivos nanoestruturados.

Atualmente um capacitor pode realizar a função de uma bateria, uma vez que é usado para armazenar cargas que são fornecidas a um circuito. A grande prerrogativa é que, independe de reações químicas diretas, o supercapacitor não irá obter um equilíbrio químico com um determinado tempo de uso, evitando assim, de ser inutilizado após sucessivas cargas e descargas, sendo esta a problemática das baterias comuns e/ou recarregáveis.

A problemática dos capacitores normais como fonte de alimentação é que à medida que o capacitor descarrega, a diferença de potencial entre seus eletrodos decresce rapidamente, devido ao equilíbrio eletrostático.

Outro malefício ainda maior é a sua capacidade de armazenamento de carga que não se assemelha com os de baterias.

Considerando a concepção de um supercapacitor como sendo um dispositivo com uma capacidade elevada para armazenamento de cargas, pode-se implicar que haverá um tempo de descarga superior aos capacitores convencionais, quando este estiver ligado a um circuito. Portanto opta-se pela redução do tamanho para que seja competitivo com as atuais baterias de íons de lítio.

Tendo inicialmente o Óxido de Ferro $\left(\mathrm{Fe}_{2} \mathrm{O}_{3}\right)$ como base de estudo e investigação, propõem-se o desenvolvimento de métodos eficazes para depor em substratos que não alterem as condições elétricas, mecânicas, químicas das NP's e eletrodos coletores de cargas auxiliados por nanotubos de carbono.

De modo igual, se visto que os possíveis substratos para suporte da nanoestrutura podem vir a auxiliar nas características de um dispositivo final, se estudará melhorias para facilitar e proporcionar um custo-benefício de produção da nanotecnologia formada. Diversos problemas de engenharia poderão ser vistos e melhorados desde a geração das nanopartículas até a construção dos possíveis dispositivos, dado as características geradas pelas NP's de $\mathrm{Fe}_{2} \mathrm{O}_{3}$ e das nanoestruturas de natureza semelhante, porém com materiais ainda não aplicados a supercapacitores, como o Paládio ( $\mathrm{Pd})$, Platina (Pt) e Cobre $(\mathrm{Cu})$.

\section{Conclusões}

Neste trabalho objetiva-se realizar estudos fundamentais e sistemáticos de nanomateriais, inicialmente a base de $\mathrm{Fe}_{2} \mathrm{O}_{3}$ em solução. Posteriormente, o uso de outros materiais ampliará significativamente o potencial para novos resultados 
qualitativos e quantitativos, não somente para os dispositivos capacitivos, mas para uma vasta gama de componentes eletrônicos.

Planejando a aplicação direta, tanto do $\mathrm{Fe}_{2} \mathrm{O}_{3}$ como do $\mathrm{Pd}$, $\mathrm{Pt}$ e $\mathrm{Cu}$ na nanofabricação de capacitores eletroquímicos no estado sólido e/ou líquido. Serão explorados os tipos de configurações para montagem dos supercapacitores, desde as configurações das nanopartículas e suas ligas metálicas como da disposição em substratos de base orgânica, poliméricas e metálicas.

\section{Referências}

BOITA, J.; NICOLAO, L.; ALVES, M. C. M.; MORAIS, J., Controlled growth of metallic copper nanoparticles. New Journal of Chemistry 2017, 41, (23), 14478-14485.

KLEM, M. d. S. estudo de supercapacitores orgânicos impressos em papel à base de pedot:pss. Universidade Paulista "Júlio de Mesquita Filho - UNESP", 2017.

MIN, J.; KIERZEK, K.; CHEN, X.; CHU, P. K.; ZHAO, X.; KALENCZUK, R. J.; TANG, T.; MIJOWSKA, E., Facile synthesis of porous iron oxide/graphene hybrid nanocomposites and potential application in electrochemical energy storage. New Journal of Chemistry 2017, 41, (22), 13553-13559.

ZARBIN, A. J. G., Química de (nano)materiais. Química Nova 2007, 30, (6), 1469 - 1479. 